\title{
La participación de las PYMES en la contratación pública: una visión desde el derecho europeo
}

\author{
BeAtriz GÓmez Fariñas ${ }^{1}$
}

\section{RESUMEN}

Las pequeñas y medianas empresas (PYMES) representan el principal motor de la economía de los distintos Estados miembros de la Unión Europea. Sin embargo, su acceso al mercado de las compras públicas se ve entorpecido por múltiples factores, entre los que destacan el gran tamaño de los contratos licitados y la exigencia de criterios de selección desproporcionados. En este trabajo se exponen las principales medidas adoptadas por las directivas europeas sobre contratación pública para fomentar la participación de estas empresas en las licitaciones públicas. En particular, centraremos nuestra atención en la división del contrato en lotes y en las limitaciones establecidas en relación con la solvencia que han de acreditar los operadores económicos.

Palabras clave: contratación pública, PYMES, discrecionalidad administrativa, división en lotes, criterios de selección.

1 Graduada en Derecho y máster en Abogacía por la Universidad de Vigo, Vigo, España Máster en Contratación Pública por la Universidad de Castilla-La Mancha, Ciudad Real, España. Actualmente es investigadora FPu en el área de Derecho Administrativo de la Universidad de Vigo, Vigo, España. Correo-e: beagomez@uvigo.es. Enlace ORCID: 00000002-8260-9527. Fecha de recepción: 28 de febrero de 2020. Fecha de modificación: 13 de marzo de 2020. Fecha de aceptación: 2 de mayo de 2020. Para citar el artículo: GÓmEZ FARIÑAS, BEATRIZ, "La participación de las PYMES en la contratación pública: una visión desde el derecho europeo", Revista digital de Derecho Administrativo, Universidad Externado de Colombia, n. ${ }^{\circ} 24,2020$, pp. 161-182. DOI: https://doi.org/10.18601/21452946.n24.07. 


\title{
SME Participation in Public Procurement: Analysis from a European Law Perspective
}

\author{
ABSTRACT
}

Small and medium-sized enterprises (SMEs) are the main engine of the economy in the various Member States of the European Union. However, their access to the public procurement market is hampered by many factors, including the large size of the contracts and the requirement for disproportionate selection criteria. This paper sets out the main measures adopted by the European Directives on public procurement to encourage the participation of these companies in public tenders. In particular, we will focus our attention on the division of the contract into lots and the limitations established in relation to the solvency to be proved by the economic operators.

Keywords: Public Procurement, SMEs, Administrative Discretion, Division into Lots, Selection Criteria.

\section{LA PARTICIPACIÓN DE LAS PYMES EN EL MERCADO EUROPEO DE LAS COMPRAS PÚBLICAS}

Desde sus inicios, la Unión Europea ha establecido como su objetivo prioritario la creación de un "mercado común" orientado a mejorar el bienestar económico y el crecimiento de la Unión en su conjunto, así como de los distintos Estados que la componen ${ }^{2}$. El sector de la contratación pública, debido fundamentalmente a su singular importancia en términos económicos ${ }^{3}$, pronto se reveló como un instrumento clave para la consecución de tan codiciado propósito ${ }^{4}$. Las instancias europeas han centrado sus esfuerzos en la apertura

2 Artículo 2 del Tratado Constitutivo de la Comunidad Económica Europea (en lo sucesivo, TCEE), firmado en Roma en 1957 y que, tras la entrada en vigor del Tratado de Lisboa en 2009, recibiría la denominación de Tratado de Funcionamiento de la Unión Europea.

3 En atención a los datos publicados por la Comisión Europea, en 2015, las compras públicas en el marco de la Unión Europea se cifraron en 2015,3 billones de euros (excluidos los servicios públicos), lo que supone un incremento del 4,2\% respecto del año anterior. Además, se estima que esta actividad representa un promedio del $13 \%$ del PIB de los distintos Estados miembros. Estos datos han sido extraídos del documento de la Comisión Europea Public Procurement Indicators 2015, del 9 de diciembre de 2016, disponible en línea: http://ec.europa.eu/DocsRoom/documents/20679/attachments/1/translations/en/ renditions/pdf [consultado el 23 de diciembre de 2019].

4 En este sentido se pronunció la Comisión en su Libro Blanco sobre la realización del mercado interior Completing the Internal Market, del 14 de junio de 1985 [COM (85) 310 final], pp. 23-24, cuyo apartado 81 resalta la importancia de este sector para alcanzar el gran objetivo de establecer un mercado común en la Unión Europea: "Public procurement covers a sizeable part of GDP and is still marked by the tendency of the authorities concerned to keep their purchases 
del mercado de las compras a la más amplia competencia y, en particular, en el fomento de la participación de las pequeñas y medianas empresas (PYMES) en las licitaciones públicas ${ }^{5}$.

La gran mayoría de las empresas que engrosan el tejido empresarial de la Unión Europea tienen la consideración de PYMES, de ahí que hayan sido bautizadas como la "espina dorsal" de la economía europea ${ }^{6}$. Su alto grado de resiliencia para adaptarse a los cambios del mercado hace que representen un importante motor para el desarrollo económico y la creación de empleo. La participación de estas empresas en las licitaciones públicas permite a las entidades contratantes aumentar considerablemente su base de proveedores y conseguir una mejor relación calidad-precio en sus adquisiciones ${ }^{7}$. Al mismo tiempo, el reparto de la cuota de mercado entre distintos operadores económicos evita el riesgo de dependencia de una determinada empresa y dificulta las prácticas colusorias que habitualmente se producen en este sector ${ }^{8}$.

Desde el punto de vista empresarial, el acceso a este mercado les brindaría una magnífica oportunidad para desarrollar su enorme potencial de crecimiento e innovación, con el consiguiente impacto positivo sobre la economía9. Estas

and contracts within their own country. This continued partitioning of individual national markets is one of the most evident barriers to the achievement of a real internal market". La Comisión volvería a insistir en esta idea dos años después, en su comunicación Vademécum sobre los contratos públicos en la Comunidad [DO C 358/1 del 31 de diciembre de 1987].

5 La Unión Europea ha acuñado una definición de PYME que se encuentra recogida en el Anexo I del Reglamento (UE) n. ${ }^{\circ}$ 651/2014 de la Comisión, del 17 de junio de 2014, por el que se declaran determinadas categorías de ayudas compatibles con el mercado interior en aplicación de los artículos 107 y 108 del Tratado [DOuE L187 del 26 de junio de 2014]. De acuerdo con el artículo 2 del citado Anexo, la categoría de microempresas, pequeñas y medianas empresas (PYMES) está constituida por las empresas que ocupan a menos de 250 personas y cuyo volumen de negocios anual no excede de 50 millones de euros o cuyo balance general anual no excede de 43 millones de euros.

6 Las pequeñas y medianas empresas constituyen el 99\% de la totalidad de las empresas que operan en la Unión Europea. En España, la situación es muy similar y el porcentaje de empresas que merecen tal consideración asciende al 99,8\%. Estos datos han sido extraídos del documento Retrato de la PYME, elaborado por el Ministerio de Industria, Economía y Competitividad, disponible en línea: http://www.ipyme.org/Publicaciones/Retrato-PYMEDIRCE-1 - enero-2017.pdf [consultado el 4 de enero de 2020].

7 José María Gimeno Feliú, "La necesidad de un código de contratos públicos en España. La contratación pública y las PYMES como estrategia de reactivación de la economía", en José María Gimeno Feliú (dir.), Observatorio de los Contratos Públicos 2011, Cizur Menor: Civitas, 2012, p. 61.

8 Martin Trybus y Marta Andrecka, "Favouring Small and Medium Sized Enterprises with Directive 2014/24/EU?", Public Procurement Law Review, n. ${ }^{\circ} 3$, 2017, pp. 226-228.

9 Véase el Libro Verde La contratación pública en la Unión Europea: reflexiones para el futuro, del 26 de noviembre de 1996 [COM (96) 583 final], p. 30. En la doctrina, el potencial innovador de las PYMES y su gran capacidad de adaptación al mercado han sido destacados -entre otros autores- por Gonzalo Barrio García, "Contratación pública y pymes. Un comentario a la luz de la propuesta de Directiva en materia de contratación pública", en J. José Pernas García (dir.), 
empresas cuentan con un alto nivel de especialización que las convierte en particularmente idóneas para concurrir a contratos que demandan una serie de conocimientos específicos, como aquellos encuadrados en el marco del sector tecnológico. Por esta razón, no es descabellado afirmar que la innovación de las compras públicas va a depender en buena medida de su apertura a las PYMES innovadoras.

Pese a todo, las PYMES tienen que sortear numerosos obstáculos para convertirse en contratistas del sector público ${ }^{10}$. Los contratos públicos suelen ser complejos y su ejecución demanda una capacidad económica y/o unas competencias profesionales que no se encuentran al alcance de cualquier organización. A menudo, las empresas de menor tamaño desconocen la existencia de licitaciones en curso o se encuentran con problemas a la hora de acceder a la información, debido, fundamentalmente, a la carencia de recursos personales suficientes destinados a este fin ${ }^{11}$. Por otro lado, la gran envergadura de los contratos, el elevado coste de preparación de las ofertas, la insuficiencia del plazo para presentarlas o la exigencia de garantías desproporcionadas son factores que desincentivan su participación. A ello hay que sumar la falta de personal capacitado para elaborar correctamente las ofertas y las dificultades para acceder a líneas de financiación.

La necesidad de tomar medidas para contrarrestar las dificultades referidas ya había sido destacada por la Comisión Europea en el Libro Verde "La contratación pública en la Unión Europea: reflexiones para el futuro" (1996) ${ }^{12}$. Con posterioridad, se reiteró la conveniencia de adaptar las compras públicas a las necesidades de este colectivo en su documento de trabajo Código Europeo de Buenas Prácticas para Facilitar el Acceso de las PYME a los Contratos Públicos ${ }^{13}$, en el

Contratación pública estratégica, Cizur Menor (Navarra): Thomson Reuters-Aranzadi, 2013, pp. 133-159; MARTIN BurGI, "Small and Medium-Sized Enterprises and Procurement Law - European Framework and German Experiences", Public Procurement Law Review, n. ${ }^{\circ} 16$, 2007, pp. 285-286; y Patricia Valcárcel FernándeZ, "Impulso de la compra pública para la innovación (CPI) a través de las distintas modalidades de contratación conjunta: análisis de casos", en Patricia Valcárcel Fernández (dir.), Compra conjunta y demanda agregada en la contratación del sector público. Un análisis jurídico y económico, Cizur Menor: Aranzadi-Thomson Reuters, 2016, pp. 361-362.

10 En 2017 solamente un 28\% de los contratistas del sector público en España eran PYMES, un porcentaje muy inferior al promedio de la Unión Europea, que se sitúa en torno al $52 \%$. Estos datos han sido extraídos del Estudio Económico de España realizado por la OCDE en noviembre de 2018, p. 61 .

11 Ministerio de Hacienda y Función Pública, Informe relativo a la contratación pública en España 2017, Gobierno de España, 2018, pp. 63-64.

12 Libro Verde "La contratación pública en la Unión Europea: reflexiones para el futuro", del 27 de noviembre de $1996[\operatorname{COM}(96) 583$ final $]$.

13 Código Europeo de Buenas Prácticas para Facilitar el Acceso de las PYME a los Contratos Públicos, del 25 de junio de 2008 [SEC(2008)2193]. 
cual se recogían una serie de orientaciones para eliminar los obstáculos con los que se encuentran a la hora de acceder al mercado.

Esta iniciativa se materializó con la aprobación de las directivas europeas sobre contratación pública en $2004^{[14]}$, que ya incorporaban algunas medidas de promoción de las PYMES, pero pronto se constató la insuficiencia de esta regulación y la necesidad de redefinir las políticas públicas perseguidas a través de este sector ${ }^{15}$. Así, el impulso decisivo tuvo lugar con la aprobación de la Directiva sobre Contratación Pública de 2014 (en adelante, DCP) ${ }^{16}$, que pretende crear un escenario más competitivo apostando por procedimientos más racionales y flexibles que fomenten la participación de estas empresas ${ }^{17}$. Además, incorpora una serie de medidas específicas claramente orientadas en esta dirección, entre las que cabe destacar las siguientes: a) la división del contrato en lotes; b) la limitación de los requisitos de solvencia que han de cumplir los operadores económicos; c) la acreditación del cumplimiento de las condiciones exigidas para acceder a la licitación a través de la presentación de una declaración responsable (Documento Europeo Único de Contratación); y d) el pago directo a los subcontratistas.

La aprobación de esta norma supone un punto de inflexión en la lucha por eliminar las desigualdades entre operadores económicos en el acceso al procedimiento de contratación. En este sentido, se aboga por la simplificación de los trámites procedimentales y la reducción de las cargas administrativas

14 Directiva 2004/17/CE del Parlamento Europeo y del Consejo, del 31 de marzo de 2004, sobre la coordinación de los procedimientos de adjudicación de contratos en los sectores del agua, de la energía, de los transportes y de los servicios postales [DOUE L134, del 30 de abril de 2004]; y Directiva 2004/18/CE del Parlamento Europeo y del Consejo, del 31 de marzo de 2004, sobre coordinación de los procedimientos de adjudicación de los contratos públicos de obras, de suministro y de servicios [DOUE L134, del 30 de abril de 2004].

15 Cristina Clementina Arenas Alegría, "Medidas para fomentar la participación de las pymes en la contratación pública. Análisis de las directivas de cuarta generación", Revista Española de Derecho Administrativo, n. ${ }^{\circ} 182,2017$, pp. 375-408.

16 Directiva 2014/24/UE del Parlamento Europeo y del Consejo, del 26 de febrero de 2014, sobre contratación pública y por la que se deroga la Directiva 2004/18/CE [DOUE L94, del 28 de marzo de 2014]. Este marco normativo se completa con la Directiva 2014/23/ UE del Parlamento Europeo y del Consejo, del 26 de febrero de 2014, relativa a la adjudicación de contratos de concesión [DOUE L94, del 28 de marzo de 2014]; y la Directiva 2014/25/UE del Parlamento Europeo y del Consejo, del 26 de febrero de 2014, relativa a la contratación por entidades que operan en los sectores del agua, la energía, los transportes y los servicios postales, y por la que se deroga la Directiva 2004/17/CE [DOuE L94, de 28 de marzo de 2014].

17 Los objetivos de esta cuarta generación de Directivas en materia de contratación pública y las vicisitudes acontecidas a lo largo de su proceso de elaboración han sido analizados -desde una perspectiva crítica- por Roberto Caranta, "The changes to the Public Contract Directives and the story they tell about how EU Law works", Common Market Law Review, n. ${ }^{\circ} 52,2015$, pp. 391-460. 
para evitar que los operadores económicos se vean excluidos de la licitación de forma injustificada ${ }^{18}$.

En España, el legislador también ha hecho eco de la necesidad de impulsar la participación de las PYMES para lograr una mejor relación calidad-precio de la prestación contractual, sin duda influenciado por la nueva normativa europea $^{19}$. La Ley de Contratos del Sector Público ${ }^{20}$ (en adelante, LCSP) aprobada en 2017 alude en su preámbulo a la promoción de las PYMES como uno de los verdaderos objetivos de la ley. Esta declaración de intenciones se concreta en el artículo 1.3 de la norma, de acuerdo con el cual "se facilitará el acceso a la contratación pública de las pequeñas y medianas empresas, así como de las empresas de economía social". Su ubicación sistemática no deja lugar a dudas acerca de la importancia que reviste este propósito, que ha de inspirar la actuación de las entidades contratantes a la hora de diseñar el procedimiento y condiciones de la contratación.

En las páginas que siguen centraremos nuestra atención en dos aspectos sumamente relevantes para lograr el objetivo de convertir el mercado de las compras públicas en un escenario más favorable para las empresas de menor dimensión. En primer término, abordaremos la configuración del objeto del contrato y, en particular, nos detendremos en la posibilidad de dividirlo en dos o más lotes diferenciados. A continuación, analizaremos las medidas introducidas por la normativa europea sobre contratación pública para evitar que estas empresas se vean sometidas a unos criterios de selección desproporcionados.

\section{LA DISCRECIONALIDAD ADMINISTRATIVA EN LA CONFIGURACIÓN DEL OBJETO CONTRACTUAL}

Uno de los principales problemas con los que se encuentran las empresas a la hora de participar en los procedimientos de contratación es el excesivo tamaño de los contratos que licitan los entes del sector público ${ }^{21}$. Esta circunstancia hace que un elevado porcentaje de operadores económicos se vea excluido

18 Martin Trybus y Marta Andrecka, óp. cit., p. 224.

19 Dolors Canals AmetLler, "Divisibilidad del objeto contractual y otras medidas legales de fomento de la participación de las pymes en la contratación pública", Cuadernos de Derecho Local, n. ${ }^{\circ} 48$, 2018, pp. 225-227; e IsAbel GAllego CórCOles, "El derecho de la contratación pública: Evolución normativa y configuración actual", en Eduardo Gamero Casado e Isabel Gallego Córcoles (dir.), Tratado de contratos del sector público, Valencia: Tirant Lo Blanch, 2018, p. 95; y José AnTONIO Moreno Molina, Una nueva contratación pública social, ambiental, eficiente, transparente y electrónica, Albacete: Bomarzo, 2018, pp. 43 y ss.

20 Ley 9/2017, del 8 de noviembre, de Contratos del Sector Público, por la que se transponen al ordenamiento jurídico español las Directivas del Parlamento Europeo y del Consejo 2014/23/UE y 2014/24/UE, del 26 de febrero de 2014.

21 Así lo ha puesto de manifiesto la Comisión en su documento de trabajo Código Europeo de Buenas Prácticas para Facilitar el Acceso de las PYME a los Contratos Públicos, óp. cit., p. 7. 
de la competición al no contar con la capacidad suficiente para presentar una oferta por la totalidad del contrato, ya sea en términos económicos o relacionados con su aptitud profesional.

Los órganos de contratación cuentan con un amplio margen de discrecionalidad a la hora de determinar el objeto del contrato en función de sus necesidades y del modo que mejor satisfaga el interés público perseguido con la contratación. La decisión de licitar un contrato público como una unidad indivisible o de fraccionarlo en varios lotes, así como de agrupar varias prestaciones en un mismo contrato, entraña un indudable componente de discrecionalidad técnica que debe atender necesariamente a las características del contrato y a la situación del mercado relevante ${ }^{22}$.

La normativa europea en materia de contratos públicos recoge diversas opciones en lo tocante al diseño del objeto contractual, de modo que las entidades del sector público pueden escoger la que resulte más procedente a la vista de los factores anteriormente señalados. Las prestaciones que pueden ser objeto de un contrato público son muy diversas, pues abarcan desde las necesidades más básicas de las entidades públicas hasta proyectos de gran complejidad y envergadura; esto hace que no exista una única solución y que la corrección de la configuración del contrato haya de ser analizada caso por caso. Tanto si el órgano de contratación opta por licitar un gran contrato para aprovecharse de las economías de escala y lograr así una mayor eficiencia del gasto público, como si decide dividirlo en porciones más pequeñas para fomentar una mayor competencia en el mercado, su decisión ha de encontrarse debidamente justificada y guardar un absoluto respeto hacia los principios generales de la contratación pública. La figura de la discrecionalidad técnica en ningún caso puede ser utilizada para burlar las exigencias de estos principios y amparar una contratación que resulte ajena al derecho a una buena administración ${ }^{23}$.

A continuación, veremos que la DCP -y por consiguiente la legislación española en esta materia- se ha inclinado decididamente a favor de la división del contrato en varios lotes diferenciados. Sin embargo, es preciso dejar sentado que esta técnica no siempre es la más adecuada y debe ser necesariamente conciliada con los mecanismos de agregación de la demanda que también han recibido un fuerte impulso a nivel europeo.

22 Juan Antonio Chinchilla Peinado, "La preparación del contrato. En especial, solvencia y criterios de adjudicación", en Silvia Díez Sastre (dir.), La nueva Ley de Contratos del Sector Público. Curso de actualización. Especial referencia a la contratación de las entidades locales, La Coruña: Iuris Utilitas, 2018, p. 86.

23 En la doctrina, Rodríguez-Arana Muñoz y Herce Maza han afirmado que "la buena administración de la compra pública encuentra su operatividad en el cumplimiento de los principios de la Unión Europea". Cfr. Jaime Rodríguez-Arana Muñoz y José Ignacio HerCe MAZA, "La buena administración en la contratación pública: mención especial a la fase de ejecución del contrato", Gabilex, n. ${ }^{\circ}$ Extra. 1, 2019, p. 31. 


\subsection{LA DIVISIÓN EN LOTES DE LOS CONTRATOS PÚBLICOS}

La división del objeto del contrato en lotes ha recibido un impulso significativo a raíz de la aprobación de la normativa europea sobre contratación pública de 2014. A pesar de que la anterior generación de directivas ya hacía alguna breve alusión a esta medida en relación con el valor estimado del contrato ${ }^{24}$ y con la información que debía incluirse en el anuncio de licitación ${ }^{25}$, lo cierto es que no regulaba con precisión las condiciones para su ejercicio. Esta situación de incertidumbre ha animado al legislador europeo a dotar a dicha figura de un marco normativo consistente que facilite su utilización por las entidades contratantes de los distintos Estados miembros ${ }^{26}$.

La DCP se pronuncia ahora de forma expresa sobre esta cuestión y establece en su artículo 46.1 que "los poderes adjudicadores podrán optar por adjudicar un contrato en forma de lotes separados, y podrán decidir el tamaño y objeto de dichos lotes". En el supuesto de que entiendan que no procede la división en lotes, deberán indicar las principales razones que han motivado su decisión. De acuerdo con esta previsión, las entidades contratantes de los distintos Estados miembros solamente se encuentran obligadas a estudiar la conveniencia de dividir los contratos que liciten, pero la decisión final sobre si hacerlo o no pertenece a su esfera de discrecionalidad. Ello sin perjuicio de la facultad de los Estados miembros de establecer la división del contrato en lotes como obligatoria, en las condiciones que se especifiquen en su normativa interna.

En España, esta posibilidad se concibió en un primer momento como una facultad excepcional en manos de los órganos de contratación, que debían justificar las razones por las cuales consideraban oportuno apartarse de la regla general de licitar el contrato en su totalidad ${ }^{27}$. Sin embargo, con la aprobación de la LCSP, este patrón se invierte y la división del contrato se convierte en la regla general. Los órganos de contratación estarán obligados a dividir el contrato en partes independientes "siempre que la naturaleza o el objeto del contrato lo permitan" (artículo 99 LCSP). Solamente podrán apartarse de este mandato en caso de que existan motivos válidos para ello, los cuales deberán

Artículo 9.5 de la Directiva 2004/18/CE.

Anexo VII A, apartado 7.

David Muñoz Zapata, "La división del contrato en lotes tras la aprobación de la cuarta generación de Directivas", Contratación Administrativa Práctica, n. ${ }^{\circ}$ 136, 2015, pp. 42-63.

Así lo establecía el artículo 86 del derogado Texto Refundido de la Ley de Contratos del Sector Público, de acuerdo con el cual el órgano de contratación podía dividir el contrato en lotes en los siguientes supuestos: a) cuando las distintas partes del contrato sean susceptibles de utilización o aprovechamiento separado y constituyan una unidad funcional b) cuando así lo exija la naturaleza del objeto; y c) cuando deban contratarse separadamente prestaciones diferenciadas dirigidas a integrarse en una obra, siempre que dichas prestaciones gocen de una sustantividad propia que permita una ejecución separada, por tener que ser realizadas por empresas que cuenten con una determinada habilitación. 
justificarse debidamente en el expediente, salvo en los casos de contratos de concesión de obras.

La propia ley recoge dos motivos que en todo caso tienen la entidad suficiente como para justificar la no división en lotes del objeto del contrato. El primero de ellos alude al hecho de que la división conlleve el riesgo de restringir injustificadamente la competencia, si bien su aplicación se encuentra supeditada a la existencia de un informe previo emitido por la autoridad en materia de defensa de la competencia. El segundo, por su parte, hace referencia a que la correcta ejecución del contrato se vea dificultada desde el punto de vista técnico, o bien porque la naturaleza del objeto demande una coordinación en la ejecución de las distintas prestaciones que podría verse imposibilitada con motivo de la adjudicación de los lotes a una pluralidad de contratistas. Es preciso señalar que esta lista no tiene carácter tasado, de modo que los órganos de contratación deberán valorar en cada caso la procedencia o no de la división.

Antes de proceder a la división del objeto contractual en varios lotes, el órgano de contratación ha de razonar acerca de la posibilidad y conveniencia de utilizar este mecanismo. La división puede realizarse de forma cuantitativa, haciendo que la magnitud de cada contrato se corresponda mejor con las capacidades de las PYMES; o en base a criterios cualitativos o de especialización ${ }^{28}$, especialmente cuando el contrato está conformado por varias prestaciones claramente diferenciadas. Esta última opción es muy interesante porque fomenta la inversión de las pequeñas empresas para lograr una mayor especialización en el sector en cuestión, al tiempo que permite a las entidades contratantes satisfacer sus necesidades con soluciones innovadoras que pueden resultar incluso más rentables que las tradicionales.

En cualquier caso, el órgano de contratación goza de libertad para determinar el número y tamaño de cada uno de los lotes del modo que estime más oportuno para la correcta satisfacción del interés general, siempre que respete los principios nucleares de la contratación pública. En particular, deben observar las exigencias del principio de proporcionalidad, que va a desempeñar un papel muy relevante en el diseño de los lotes. La confección de lotes demasiado grandes no cumple con el objetivo de facilitar la participación de las PYMES en los procedimientos de contratación. Tampoco es conveniente fraccionar el contrato en un número de lotes excesivo o de un tamaño insignificante, pues ello podría dificultar su gestión separada e incluso conducir a una contratación ineficiente. Es necesario, pues, calibrar debidamente su tamaño para lograr

28 Véase el considerando 78 de la Directiva 2014/24/UE. En la doctrina, MARía Del CaRmEN Rodríguez Martín-Retortillo, "Fraccionamiento del contrato y división en lotes. Posibilidad de limitar el número de lotes a los que un operador económico pueda licitar o adjudicarse a cada licitador", Anuario da Facultade de Dereito da Universidade da Coruña, vol. 21, 2017, pp. 221-222. 
un equilibrio entre la apertura del mercado a la competencia y la eficiencia de la contratación ${ }^{29}$.

\subsubsection{La obligación de justificar la no división del contrato en lotes}

La DCP establece una obligación prima facie de dividir el objeto del contrato público que va a ser licitado en varios lotes de menor tamaño, especialmente cuando se trata de grandes contratos ${ }^{30}$. Si el órgano de contratación considera que dicha división no es conveniente, debe exteriorizar las razones que lo han llevado a tomar tal decisión y dejar constancia de las mismas en el expediente de contratación. Esta posibilidad de apartarse de la regla general cuando existan razones de peso que avalen la contratación integral de la prestación se conoce con el nombre de "divide or explain" (dividir o explicar). Este principio pretende encauzar el comportamiento de las entidades contratantes en una determinada dirección, pero concediéndoles al propio tiempo una mayor flexibilidad en su actuación a la vista de las circunstancias del caso.

Dicho lo anterior, surge el interrogante de hasta donde se extiende la carga argumentativa que deben soportar los órganos de contratación. Esta cuestión no ha sido pacífica y ha sufrido importantes variaciones durante los trabajos preparatorios de la normativa europea, que ha ido rebajando el umbral de motivación que debían alcanzar los órganos de contratación hasta el punto de exigir simplemente que se indiquen las "principales razones" por las cuales han decidido no subdividir el contrato en lotes ${ }^{31}$. La citada previsión contrasta notablemente con lo inicialmente dispuesto en la Propuesta de Directiva, de acuerdo con la cual debían proporcionar una "explicación específica" de sus motivos en el anuncio de licitación o en la invitación a confirmar el interés ${ }^{32}$.

29 En la misma línea se pronuncia MARTIN TRYBus, "The division of public contracts into lots under Directive 2014/24: minimum harmonisation and impact on SMEs in public procurement?", European Law Review, vol. 43, n. ${ }^{\circ} 3$, 2018, pp. 326-327. Este autor señala que en la determinación del número y tamaño de los lotes ha de tomarse en consideración la estructura del mercado con sus consiguientes fluctuaciones, que va a depender en buena medida del número de licitadores potenciales y de su comportamiento, pues en caso contrario se estaría comprometiendo la competencia y la relación calidad-precio de las ofertas.

30 Miguel Assis Raimundo, "Aiming at the market you want: a critical analysis of the duties on division into lots under Directive 2014/24/EU", Public Procurement Law Review, n. ${ }^{\circ} 167$, 2018, pp. 175-177.

31 Como ya se ha indicado, esta previsión se recoge en el artículo 46.1, párrafo segundo, de la Directiva 2014/24/UE.

32 Artículo 44.1 de la Propuesta de Directiva del Parlamento Europeo y del Consejo relativa a la contratación pública, del 20 de diciembre de 2011 [СОм(2011) 896 final]. 
En este sentido, cabe poner de relieve que la formulación de esta obligación que finalmente ha sido recogida en la Directiva sobre contratación pública resulta demasiado laxa y puede dar pie a que los órganos de contratación la asuman como un mero formalismo, que puede ser fácilmente cumplimentado aportando una justificación genérica o estandarizada ${ }^{33}$. Además, salta a la vista que la ausencia de una explicación consistente no se compadece bien con el derecho a una buena administración que asiste a los operadores económicos y constriñe notablemente sus posibilidades de impugnar con éxito la decisión del órgano de contratación.

Esta disfunción de la normativa europea ha sido correctamente encauzada por el legislador español que, como vimos, solamente faculta al órgano de contratación para sustraerse de la obligación de dividir el objeto contractual en lotes cuando: a) concurra un motivo válido para ello $;$ y b) esta circunstancia se justifique debidamente en el expediente de contratación. Pero, además, tal justificación ha de ser razonable y suficiente, de modo que se detallen cuáles son los efectos perniciosos que desaconsejan -desde el punto de vista técnico o económico- la división en lotes.

Dado que la división en lotes constituye ahora la norma general, si las entidades contratantes deciden no seguirla y optan en su lugar por la licitación conjunta de la prestación, deberán invocar otros intereses que, en el supuesto concreto, gocen de prevalencia frente al objetivo de fomentar la competencia en el mercado. No basta con que se haga referencia explícita a la causa que permite excepcionar dicha norma, sino que es necesario que su concurrencia quede debidamente acreditada ${ }^{34}$. En caso contrario, entiendo que lo procedente sería la anulación de los pliegos del contrato, sin que la ausencia de justificación pueda ser suplida a posteriori por el órgano de contratación. Los operadores económicos que tengan interés en participar en la licitación deben conocer de antemano las razones que decantan la balanza a favor de la no división, por cuanto tal información será determinante para animarlos o no a participar en la competición y, en su caso, impugnar la validez de las mismas cuando todavía están a tiempo para hacerlo.

33 La doctrina ha venido advirtiendo del riesgo de que los órganos de contratación recurran a un "copia y pega" de las razones que motivan la no división del objeto del contrato. En este sentido, Ignacio Herrera AnChustegui, "Division into lots and demand aggregation -extremes looking for the correct balance", en Grith Skovgaard Ølykke y Albert Sánchez-Graells (dirs.), Reformation or Deformation of the EU Public Procurement Rules, Cheltenham: Edward Elgar Publishing, 2016, pp. 133-135; y MARTIN TRYBus, "The promotion of Small and Medium Sized Enterprises in Public Procurement: A Strategic Objective of the New Public Sector Directive?", en Francois Lichere, Roberto Caranta y Steen Treumer (eds.), Modernising public procurement: the new Directive, Copenhagen: DJøF Publishing, 2014, p. 266. 


\subsubsection{Limitaciones en la presentación de ofertas y la adjudicación de los lotes}

A pesar de su indudable relevancia como medida de fomento de la participación de las PYMES en el mercado de las compras públicas, la división del contrato en lotes no es suficiente por sí sola para alcanzar este objetivo. Puede suceder que un operador económico que goce de una solvencia amplia decida presentar oferta a los distintos lotes y resulte adjudicatario de todos ellos, o de gran parte de los mismos ${ }^{35}$. En este supuesto, la partición del objeto contractual resultaría infructuosa, por cuando el resultado sería el mismo que si el contrato se hubiese licitado de forma unitaria: su adjudicación a un mismo licitador. Para evitar esta situación, los órganos de contratación se encuentran facultados para introducir una serie de limitaciones en relación con el número de lotes para los que un mismo operador económico puede presentar ofertas (artículo 99.4 LCSP). También podrán limitar el número máximo de lotes que pueden ser adjudicados a un mismo candidato o licitador.

Si deciden imponer alguna de estas limitaciones, deberán indicarlo expresamente en el anuncio de licitación y en el pliego de cláusulas administrativas particulares, dando cuenta de las razones que motivan su decisión en el expediente de contratación. Los pliegos del contrato han de establecer los criterios o normas que serán utilizados para el caso de que, una vez aplicados los criterios de adjudicación, un licitador pueda resultar adjudicatario de un número de lotes que exceda del máximo indicado en el anuncio de licitación y en el pliego por haber presentado las ofertas más ventajosas. Estos criterios podrán ser determinados libremente por el órgano de contratación, siempre que sean objetivos y no discriminatorios ${ }^{36}$.

Como avanzamos, estas limitaciones tienen por objeto asegurar un reparto de los distintos lotes entre varios operadores económicos y evitar que todos ellos caigan en manos de una misma empresa que, gracias a su mayor tamaño, se encuentra en una mejor posición para presentar ofertas competitivas. Sin embargo, no dejan de ser controvertidas por cuanto ponen en entredicho la efectividad de los principios de competencia y eficiencia, especialmente cuando lo que se limita es la presentación de ofertas. No hay que perder de vista que en ese caso lo que realmente se está constriñendo es el derecho de los

35 Francisco Blanco López, "División del objeto contractual en lotes. Delimitación de los lotes a los que se puede licitar y de los que se puede resultar adjudicatario. Medidas a favor de las PYME", Observatorio de Contratación Pública, 2013. Disponible en línea: http://www.obcp. es/opiniones/division-del-objeto-contractual-en-lotes-delimitacion-de-los-lotes-los-quese-puede [consultado el 5 de noviembre de 2019].

36 Sobre los distintos aspectos a tener en cuenta en la adjudicación de los lotes, véase la Nota Informativa 2/2014, del 9 de mayo, de la Secretaría Técnica de la Junta Consultiva de Contratación Administrativa de la Generalitat de Cataluña. 
operadores económicos a participar en la licitación, de modo que se verían impedidos a licitar por una parte del contrato, aunque gocen de la capacidad necesaria e incluso sean la mejor opción para satisfacer las necesidades de la entidad contratante. En este sentido, los órganos de contratación deben evitar que se produzca una alteración de las condiciones normales de competencia, siendo preferible hacer un diseño de los lotes más ajustado a las necesidades de las pequeñas empresas, pero sin disuadir a los contratistas habituales ni desincentivar el crecimiento empresarial ${ }^{37}$.

Por otro lado, la limitación del número de lotes que pueden adjudicarse a un mismo licitador supone una excepción a la obligación de contratar con la empresa que presente la oferta más ventajosa ${ }^{38}$. A pesar de que en principio esta medida es menos lesiva para los derechos de los operadores económicos, lo cierto es que puede conducir a una merma de la eficiencia de la contratación, por cuanto los lotes no siempre serán adjudicados a aquella empresa que haya presentado la oferta más ventajosa. Por esta razón, será preciso valorar en cada caso concreto qué interés ha de prevalecer por llevar aparejados más beneficios para el interés general y, con base en este ejercicio de ponderación, decidir si se establece o no tal limitación y en qué términos.

\subsubsection{La posibilidad de adjudicar varios lotes a una oferta integradora}

La principal dificultad que presenta la división del contrato en lotes es la pérdida de eficiencia que en ocasiones lleva aparejada en comparación con la licitación unitaria de la prestación. Consciente de las repercusiones que esto puede tener sobre los caudales públicos, y sin querer desatender las necesidades de las PYMES, el legislador español contempla en el artículo 99.5 LCSP la posibilidad de que los operadores económicos presenten una oferta integradora de varios lotes o incluso de la totalidad de los mismos, siempre que se permita la adjudicación de más de un lote a un mismo licitador. De este modo los órganos de contratación se encuentran en una mejor posición para seleccionar la oferta que resulte más beneficiosa para los intereses públicos, sin que el derecho de acceso de los licitadores se vea limitado por la elevada cuantía del contrato. La ocasión de competir por la adjudicación de un conjunto de lotes anima a las empresas a presentar ofertas más competitivas que en el supuesto de tener que licitar a cada uno de ellos individualmente, pues en tal caso podrán aprovecharse de las economías de escala y sacar una mayor rentabilidad de la ejecución del contrato.

37 Esta cautela ya había sido advertida por la Comisión en el Código Europeo de Buenas Prácticas para Facilitar el Acceso de las PYME a los Contratos Públicos, óp. cit., p. 7.

38 Sue Arrowsmith, The Law of Public and Utilities Procurement, vol. II, 3. ${ }^{\text {a }}$ ed., Londres: Sweet \& Maxwell, 2018, pp. 782-784. 
La utilización de este mecanismo se encuentra sujeta al cumplimiento de los requisitos que a continuación se detallan:

a. La posibilidad de presentar una oferta integradora debe advertirse en los pliegos del contrato y en el anuncio de licitación, concretando la combinación o combinaciones de lotes admitidas y la solvencia y capacidad exigida en relación con cada una de ellas.

b. La existencia de varios criterios de adjudicación.

c. La realización previa de una evaluación comparativa para determinar si la oferta integradora presentada por un licitador concreto para una combinación de lotes cumple mejor, en conjunto, los criterios de adjudicación establecidos en el pliego que las ofertas presentadas individualmente para cada uno de los lotes $^{39}$.

d. La acreditación de que se reúne la solvencia económica o financiera y técnica y profesional necesaria para la ejecución del conjunto de lotes en cuestión o, en su caso, la clasificación correspondiente.

Con todo, es preciso señalar que la formulación de una oferta integradora es potestativa para los operadores económicos, los cuales podrán limitarse a presentar ofertas a uno o varios lotes individualmente considerados si así lo estiman conveniente. Esto implica que el órgano de contratación no podrá exigir a los candidatos o licitadores que, además de licitar individualmente a varios de los lotes propuestos, presenten una oferta para cada una de las combinaciones de lotes o incluso para la totalidad del contrato. Dicho requerimiento resultaría a todas luces desproporcionado, por cuanto favorecería de forma injustificada a las empresas con mayores recursos y desvirtuaría la razón de ser de la división en lotes.

\subsection{LA AGREGACIÓN DE LA DEMANDA EN LAS COMPRAS PÚBLICAS:}

UNA NUEVA BARRERA PARA LAS PYMES

La utilización de técnicas de agregación de la demanda como vía para canalizar las contrataciones del sector público ha ido ganando un notable protagonismo en los últimos años ${ }^{40}$. Cada vez son más los compradores públicos que apuestan por comprar de forma conjunta o centralizar sus adquisiciones a través de órganos especializados, buscando una mayor profesionalización

39 Informe 11/2013, del 22 de mayo de 2013, de la Junta Consultiva de Contratación Administrativa de Aragón (Consideración Jurídica Cuarta).

40 Esta tendencia ha sido destacada por la DCP en su considerando 59: "En los mercados de contratación pública de la Unión Europea se comienza a observar una marcada tendencia a la agregación de la demanda por los compradores públicos con el fin de obtener economías de escala, incluida la reducción de los precios y de los costes de transacción, y de mejorar y profesionalizar la gestión de la contratación". 
de la contratación pública y el aprovechamiento de economías de escala ${ }^{41}$. Este mecanismo permite lograr una mayor agilidad y simplificación de los procedimientos administrativos, al tiempo que reduce los costes inherentes a la contratación.

Junto a la compra centralizada y la contratación conjunta -reguladas en los artículos 37 y 38 de la DCP-, la normativa europea regula otras técnicas de racionalización destinadas a dotar a este sector de una mayor eficacia. La primera de estas técnicas son los acuerdos marco, que pueden definirse como acuerdos celebrados entre una o varias entidades contratantes y uno o varios operadores económicos con la finalidad de establecer los términos que van a regir los contratos que se vayan a adjudicar durante un periodo de tiempo determinado (artículo 33 DCP). Estas condiciones se referirán, en particular, a los precios y a las cantidades previstas.

Por otro lado, el artículo 34 de la DCP hace referencia a los sistemas dinámicos de adquisición. Estos sistemas presentan marcadas similitudes con los acuerdos marco, pues en ambos casos se pretende comprar a gran escala para ahorrar costes y lograr así una mayor eficiencia en la utilización de los fondos públicos. Ahora bien, mientras que en el caso de los acuerdos marco el mercado permanece cerrado mientras se encuentran vigentes, los sistemas dinámicos de adquisición están permanentemente abiertos a todos aquellos operadores económicos que cumplan con los requisitos de aptitud y deseen participar en los mismos.

Resulta indubitado que la utilización de los mecanismos anteriormente referidos conlleva numerosas ventajas para los órganos de contratación, especialmente para aquellos de menor tamaño o con escasez de recursos, pero no se pueden ignorar los efectos negativos que producen sobre la competencia en el mercado ${ }^{42}$. Esta circunstancia ha sido expresamente reconocida por la DCP en su considerando 59, que pone de relieve la necesidad de supervisar con cautela su utilización para evitar que las posibilidades de acceso al mercado de las PYMES se vean mermadas. Es evidente que los órganos de contratación que se decanten por comprar a gran escala no tendrán incentivos para dividir el contrato en lotes, ya que ello les impediría alcanzar el principal objetivo que

41 Rafael Fernández AceVedo, "Aspectos jurídicos de las técnicas e instrumentos para la contratación pública agregada", en Patricia Valcárcel Fernández (coord.), Compra conjunta y demanda agregada en la contratación del sector público: un análisis jurídico y económico, Cizur Menor: Aranzadi-Thomson Reuters, 2016, pp. 31-66; y CARINA Risvig HameR, "Regular purchases and aggregated procurement: the changes in the new Public Procurement Directive regarding framework agreements, dynamic purchasing systems and central purchasing bodies", Public Procurement Law Review, n. ${ }^{\circ}$ 4, 2014, pp. 207-208.

42 Albert Sánchez Graells, Public Procurement and the EU Competition Rules, 2. ${ }^{\text {a }}$ ed., Oxford: Hart Publishing, 2015, pp. 256 y ss. 
se persigue con la agregación de la demanda: una mayor eficiencia y eficacia de la contratación motivada por la oferta de precios más competitivos ${ }^{43}$.

La normativa europea sobre contratación pública no se pronuncia con claridad sobre cuál de estas medidas ha de prevalecer con carácter general, ni establece ningún mecanismo destinado a conciliar los principios de competencia y de eficacia en la utilización de los fondos públicos. Antes bien, deja en manos de los Estados miembros la tarea de diseñar una estrategia para alcanzar un equilibrio entre ambas modalidades de compra. Para ello, es necesario que verifique si la decisión de acudir a la agregación de la demanda respeta las exigencias derivadas del principio de proporcionalidad o si, por el contrario, los efectos restrictivos de la competencia que lleva aparejados resultan excesivos a la luz del objetivo perseguido. Este ejercicio presupone la realización de una ponderación conjunta entre los principios de eficacia y eficiencia-especialmente en lo tocante al manejo de los fondos públicos-y los principios de libertad de acceso a las licitaciones, no discriminación y competencia.

El legislador español, plenamente respetuoso con la discrecionalidad de la que disfrutan los órganos de contratación para configurar el objeto del contrato, deja que sean estos los que decidan cuál es la vía que mejor permite satisfacer los intereses generales. Sin embargo, la regulación contenida en la LCSP deja entrever una clara inclinación a favor de un aumento de la participación de las PYMES. La prueba más evidente de esta tendencia es la previsión de la división del contrato en lotes como obligatoria, con la ya mencionada salvedad de que existan razones de peso que justifiquen la licitación del contrato como una unidad indivisible.

\section{NUEVAS MEDIDAS PARA FACILITAR LA PARTICIPACIÓN DE LAS PYMES: LA LIMITACIÓN DE LAS EXIGENCIAS DE SOLVENCIA}

El excesivo tamaño de los contratos licitados por el sector público no es el único problema con el que se encuentran las PYMES. Otro de los obstáculos que habitualmente han de superar para poder acceder a este mercado es la exigencia de una solvencia excesiva, es decir, de unos requisitos de aptitud tan elevados que solamente se encuentran al alcance de las grandes empresas. Los criterios de selección de licitadores tienen por objeto verificar que los candidatos o licitadores tienen capacidad económica y financiera, y que reúnen las competencias técnicas y/o profesionales necesarias para llevar el contrato a buen término (artículo 58 DCP). El cumplimiento del estándar mínimo exigido por

43 Marta Fana y Gustavo Piga, "sme's and Public Contracts. An EU Based Perspective", en Grith Skovgaard Ølykke, Carina Risvig Hansen y Christina D. Tvarnø (eds.), EU Public Directives-Modernisation, Growth and Innovation, Denmark: DJøF Publishing, 2012, pp. 37-38; e Ignacio Herrera Anchustegui, óp. cit., pp. 142-143. 
la entidad contratante es un requisito indispensable para poder participar en el procedimiento de contratación, de modo que si no se alcanza, el operador económico no podrá competir por la adjudicación del contrato ${ }^{44}$.

El carácter eliminatorio de este trámite implica que la solvencia exigida no podrá exceder de lo estrictamente necesario para garantizar la correcta realización de la prestación por el contratista, pues en caso contrario el derecho de los operadores a participar en las licitaciones públicas se vería limitado de forma desproporcionada. El establecimiento por el órgano de contratación de criterios de selección específicos ha de ir precedido de un análisis de las características del contrato, de forma que se garantice que están directamente vinculados con su objeto y que son proporcionales al mismo. En este sentido, cuanto mayor sea el tamaño del contrato, mayor será también la aptitud que han de acreditar los operadores económicos.

La DCP incorpora dos medidas novedosas que están destinadas a poner coto a la discrecionalidad de la que gozan las entidades del sector público a la hora de fijar los criterios de selección de licitadores. La primera de ellas hace referencia al volumen de negocios mínimo anual que deben reunir los operadores económicos para demostrar su solvencia económica y financiera. Pues bien, el artículo 58.3 establece que, en el supuesto de que la entidad contratante considere procedente exigir un determinado volumen de negocios, este no podrá exceder del doble del valor estimado del contrato, excepto en casos debidamente justificados en atención a los especiales riesgos que conlleva su ejecución.

Por su parte, el legislador español constriñe aún más este umbral en aras de garantizar una aplicación efectiva del principio de proporcionalidad. De acuerdo con el artículo 87.1 (a) de la LCSP, el volumen de negocios que han de acreditar los operadores económicos no podrá exceder de una vez y media el valor estimado del contrato. Cuando el contrato se divida en lotes, este criterio se aplicará en relación con cada uno de los lotes. En el supuesto de que se adjudiquen a un mismo operador económico varios lotes que deban ser ejecutados de forma simultánea, cabe la posibilidad de establecer el volumen de negocios mínimo anual por referencia a grupos de lotes. Asimismo, como sucede en el caso de la normativa europea, el citado precepto recoge

44 Julia García Santos y Juan Carlos Jurado Jiménez, "Aptitud para contratar con el sector público. Capacidad y solvencia del empresario", en Eduardo Gamero Casado e Isabel Gallego Córcoles (dirs.), Tratado de contratos del sector público, Valencia: Tirant Lo Blanch, 2018, pp. 1042-1043; y José María Gimeno Feliú, La Ley de Contratos de Sector Público 9/2017. Sus principales novedades, los problemas interpretativos y las posibles soluciones, Cizur Menor: Thomson Reuters-Aranzadi, 2019, p. 116. Por su parte, la Junta Consultiva de Contratación Administrativa, en su informe 36/07 del 5 de julio de 2007, ha definido la solvencia como "un soporte fundamental del sistema de selección del candidato a la adjudicación del contrato que permite identificar cuáles son las empresas idóneas, constituyendo el acierto en su determinación y, en su aplicación, un importante beneficio para el órgano de contratación". 
la posibilidad de que esta regla general pueda ser desplazada cuando existan razones de peso que lo justifiquen.

Por otro lado, la normativa europea faculta a los órganos de contratación para que exijan a los operadores económicos la acreditación de un nivel suficiente de experiencia demostrada mediante referencias adecuadas de contratos ejecutados en el pasado (artículo 58.4 DCP). No se puede desconocer, sin embargo, que la exigencia de una experiencia mínima en el sector constituye una importante barrera de acceso al mercado de las compras públicas. De ahí que la propia DCP limite el período temporal que va a ser tomado en consideración para valorar esta circunstancia a los últimos cinco años cuando se trata de contratos de obras, y a los últimos tres años en el caso de contratos de suministro y servicios ${ }^{45}$. Ello sin perjuicio de que, cuando sea necesario para garantizar un nivel adecuado de competencia, se puedan tener en cuenta las pruebas de las obras, suministros o servicios efectuados durante un período superior al anteriormente referido.

La LCSP regula la experiencia que han de reunir los empresarios para poder participar en la licitación de un contrato público en términos muy similares a los previstos en la DCP. En el caso de los contratos de obras, esta circunstancia ha de ser probada mediante la relación de las obras ejecutadas en el curso de los últimos cinco años (artículo 88.1 (a)), mientras que en los contratos de suministro y servicios el plazo máximo es de tres años (artículos 89.1 (a) y 90.1 (a)).

Con todo, es preciso matizar que tanto el número de referencias solicitadas como el importe de las mismas no deben ir más allá de lo estrictamente necesario para garantizar la aptitud del eventual contratista, so pena de que esta decisión sea calificada como desproporcionada. Resulta indispensable, pues, alcanzar un equilibrio entre la necesidad de las entidades contratantes de garantizar que la contratación se realiza con empresarios que cuentan con la aptitud suficiente para llevar el contrato a buen término, y el derecho de los operadores económicos a tomar parte en el procedimiento de contratación.

\section{CONCLUSIONES}

La apertura del mercado de las compras públicas a las PYMES ha sido -y todavía es a día de hoy- una de las principales tareas pendientes tanto a nivel europeo como en gran parte de los Estados miembros. No cabe duda de que las medidas legislativas implementadas a raíz de la aprobación de la cuarta generación de directivas sobre contratación pública en 2014 han supuesto un importante avance en esta dirección, pero lo cierto es que aún queda mucho camino por recorrer. La consecución de un modelo de compras públicas eficiente e innovador demanda adoptar medidas efectivas para remover los diversos obstáculos 
con los que se encuentran estas empresas en el acceso a las licitaciones públicas. Para ello, es necesario apostar por un marco normativo simple y coherente que tenga en cuenta sus necesidades sin descuidar el principal objetivo de la contratación, es decir, la adquisición de los bienes o servicios necesarios en las mejores condiciones posibles.

El éxito de las políticas de estímulo de la participación de las PYMES en las licitaciones públicas exige un cambio de mentalidad en el sector público. El nuevo paradigma de la contratación pública -y del derecho administrativo en general- aconseja dejar atrás la visión cortoplacista de las compras públicas y apostar por soluciones que sean eficientes a medio o largo plazo, en la convicción de que ello va a derivar en una mejor satisfacción de los intereses de la sociedad en su conjunto. Las entidades del sector público no pueden caer en el equívoco de observar la división del contrato en lotes como una carga adicional que han de soportar, sino como una apuesta a largo plazo por una contratación más eficiente y competitiva.

Asimismo, los órganos de contratación han de ser sumamente cautelosos a la hora de determinar las condiciones de solvencia que se van a exigir a los operadores económicos. En concreto, han de prestar una especial atención a las exigencias derivadas de la aplicación del principio de proporcionalidad, que acota tanto el volumen de negocios anual mínimo que se puede exigir como solvencia económica y financiera, como el período temporal que se tomará como referencia para valorar la experiencia del empresario a efectos de probar su solvencia técnica. La exigencia de criterios de selección desproporcionados supondría, a la postre, una limitación injustificada de la competencia en el mercado.

Finalmente, es preciso señalar que esta tendencia a favor de las empresas de menor dimensión no persigue garantizar su participación a toda costa, ni otorgarles ningún tipo de ventaja frente a las grandes empresas. Simplemente se pretende que todos los operadores económicos que cuenten con la aptitud necesaria para participar en la licitación de un contrato público puedan hacerlo en igualdad de condiciones, sin que sus derechos y/o intereses legítimos se vean afectados en exceso por la actuación administrativa.

\section{BIBLIOGRAFÍA}

Arenas Alegría, Cristina Clementina. "Medidas para fomentar la participación de las pymes en la contratación pública. Análisis de las directivas de cuarta generación". Revista Española de Derecho Administrativo, n. ${ }^{\circ} 182$, 2017, pp. 375-408.

Arrowsmith, Sue. The Law of Public and Utilities Procurement, vol. II, 3. ${ }^{a}$ ed. Londres: Sweet \& Maxwell, 2018 
Assis Raimundo, Miguel. "Aiming at the market you want: a critical analysis of the duties on division into lots under Directive 2014/24/EU". Public Procurement Law Review, n. ${ }^{\circ} 167,2018$, pp. 167-187.

Barrio García, Gonzalo. "Contratación pública y pymes. Un comentario a la luz de la propuesta de Directiva en materia de contratación pública". En J. José Pernas García (dir.), Contratación pública estratégica (pp. 133-159). Cizur Menor: Thomson Reuters-Aranzadi, 2013.

Blanco López, Francisco. "División del objeto contractual en lotes. Delimitación de los lotes a los que se puede licitar y de los que se puede resultar adjudicatario. Medidas a favor de las PYME". Observatorio de Contratación Pública, 2013. Disponible en línea: http://www.obcp.es/opiniones/division-del-objeto-contractual-en-lotesdelimitacion-de-los-lotes-los-que-se-puede [consultado el 5 de noviembre de 2019].

Burgi, Martin. "Small and Medium-Sized Enterprises and Procurement Law European Framework and German Experiences". Public Procurement Law Review, n. ${ }^{\circ} 16,2007$, pp. 284-294.

Canals Ametller, Dolors. "Divisibilidad del objeto contractual y otras medidas legales de fomento de la participación de las pymes en la contratación pública". Cuadernos de Derecho Local, n. ${ }^{\circ} 48,2018$, pp. 221-246.

Caranta, Roberto. "The changes to the Public Contract Directives and the story they tell about how EU Law works". Common Market Law Review, n. ${ }^{\circ}$ 52, 2015, pp. 391-460.

Chinchilla Peinado, Juan Antonio. "La preparación del contrato. En especial, solvencia y criterios de adjudicación". En Silvia Díez Sastre (dir.), La nueva Ley de Contratos del Sector Público. Curso de actualización. Especial referencia a la contratación de las entidades locales (pp. 128-169). La Coruña: Iuris Utilitas, 2018.

Fana, Marta, Piga, Gustavo. "Sme's and Public Contracts. An EU Based Perspective". En Grith Skovgaard Ølykke, Carina Risvig Hansen y Christina D. Tvarnø (eds.), EU Public Directives - Modernisation, Growth and Innovation (pp. 33-55). Denmark: DJøF Publishing, 2012.

Fernández AceVedo, Rafael. "Aspectos jurídicos de las técnicas e instrumentos para la contratación pública agregada". En Patricia Valcárcel Fernández (coord.), Compra conjunta y demanda agregada en la contratación del sector público: un análisis jurídico y económico (pp. 31-66). Cizur Menor: Aranzadi-Thomson Reuters, 2016.

Gallego Córcoles, IsABel. "El derecho de la contratación pública: Evolución normativa y configuración actual". En Eduardo Gamero Casado e Isabel Gallego Córcoles (dirs.), Tratado de contratos del sector público (pp. 72-160). Valencia: Tirant Lo Blanch, 2018. 
García Santos, Julia, Jurado Jiménez, Juan Carlos. "Aptitud para contratar con el sector público. Capacidad y solvencia del empresario". En Eduardo Gamero Casado e Isabel Gallego Córcoles (dirs.), Tratado de contratos del sector público (pp. 1017-1091). Valencia: Tirant Lo Blanch, 2018.

Gimeno Feliú, José María. La Ley de Contratos de Sector Público 9/2017. Sus principales novedades, los problemas interpretativos y las posibles soluciones. Cizur Menor (Navarra): Thomson Reuters-Aranzadi, 2019.

Gimeno Feliú, José María. "La necesidad de un código de contratos públicos en España. La contratación pública y las PYMES como estrategia de reactivación de la economía". En José María Gimeno Feliú (dir.), Observatorio de los Contratos Públicos 2011 (pp. 27-84). Cizur Menor: Civitas, 2012.

Herrera Anchustegui, Ignacio. "Division into lots and demand aggregation -extremes looking for the correct balance". En Grith Skovgaard Ølykke y Albert Sánchez-Graells (dir.), Reformation or Deformation of the EU Public Procurement Rules (pp. 133-135). Cheltenham: Edward Elgar Publishing, 2016.

Moreno Molina, José Antonio. Una nueva contratación pública social, ambiental, eficiente, transparente y electrónica. Albacete: Bomarzo, 2018.

Muñoz ZaPATA, DAvid. "La división del contrato en lotes tras la aprobación de la cuarta generación de Directivas". Contratación Administrativa Práctica, n. ${ }^{\circ}$ 136, 2015, pp. $42-63$.

Risvig Hamer, CARinA. "Regular purchases and aggregated procurement: the changes in the new Public Procurement Directive regarding framework agreements, dynamic purchasing systems and central purchasing bodies". Public Procurement Law Review, n. ${ }^{\circ}$, 2014, pp. 201-210.

Rodríguez-Arana Muñoz, Jaime, Herce MaZA, José Ignacio. "La buena administración en la contratación pública: mención especial a la fase de ejecución del contrato". Gabilex, n. ${ }^{\circ}$ Extra. 1, 2019, pp. 35-60.

Rodríguez Martín-Retortillo, María del Carmen. "Fraccionamiento del contrato y división en lotes. Posibilidad de limitar el número de lotes a los que un operador económico pueda licitar o adjudicarse a cada licitador". Anuario da Facultade de Dereito da Universidade da Coruña, vol. 21, 2017, pp. 192-228.

Sánchez Graells, Albert. Public Procurement and the EU Competition Rules, 2. ${ }^{a}$ ed. Oxford: Hart Publishing, 2015.

TRYbus, MARTIN. "The division of public contracts into lots under Directive 2014/24: minimum harmonisation and impact on SMEs in public procurement?". European Law Review, vol. 43, n. ${ }^{\circ}$ 3, 2018, pp. 313-342. 
Trybus, Martin. "The promotion of Small and Medium Sized Enterprises in Public Procurement: A Strategic Objective of the New Public Sector Directive?". En Francois Lichere, Roberto Caranta y Steen Treumer (eds.), Modernising public procurement: the new Directive (pp. 255-280). Copenhagen: DృøF Publishing, 2014.

Trybus, Martin, Andrecka, Marta. "Favouring Small and Medium Sized Enterprises with Directive 2014/24/EU?". Public Procurement Law Review, n. 3 3, 2017, pp. 224-238.

Valcárcel Fernández, PAtricia. "Impulso de la compra pública para la innovación (CPI) a través de las distintas modalidades de contratación conjunta: análisis de casos". En Patricia Valcárcel Fernández (dir.), Compra conjunta y demanda agregada en la contratación del sector público. Un análisis jurídico y económico (pp. 349-372). Cizur Menor: Aranzadi-Thomson Reuters, 2016. 